\title{
Renormalization Group Flows on Line Defects
}

\author{
Gabriel Cuomo ${ }^{1,2, *}$ Zohar Komargodski, ${ }^{1,2, \dagger}$ and Avia Raviv-Moshe $\odot^{1,+}$ \\ ${ }^{1}$ Simons Center for Geometry and Physics, SUNY, Stony Brook, New York 11794, USA \\ ${ }^{2}$ C. N. Yang Institute for Theoretical Physics, Stony Brook University, Stony Brook, New York 11794, USA
}

(Received 23 August 2021; revised 31 October 2021; accepted 22 December 2021; published 11 January 2022)

\begin{abstract}
We consider line defects in $d$-dimensional conformal field theories (CFTs). The ambient CFT places nontrivial constraints on renormalization group (RG) flows on such line defects. We show that the flow on line defects is consequently irreversible and furthermore a canonical decreasing entropy function exists. This construction generalizes the $g$ theorem to line defects in arbitrary dimensions. We demonstrate our results in a flow between Wilson loops in four dimensions.
\end{abstract}

DOI: 10.1103/PhysRevLett.128.021603

Introduction.-In lattice systems, in order to understand the physics on different length scales, we perform blockspin transformations, eliminating degrees of freedom that live at short distances. This process obviously reduces the overall number of degrees of freedom. But one can ask whether this reduces the number of degrees of freedom per lattice site, which is much less clear. In quantum field theory, the number of degrees of freedom per lattice site is roughly speaking the number of fields and this raises the question of whether the number of fields decreases as we probe physics of longer and longer distances.

To address these questions precisely one has to give a nonperturbative definition of what the "number of fields" means and provide a prescription to evaluate it even when there is no weakly coupled description in terms of fields. Starting from the work of Zamolodchikov on the $c$ function in two dimensions [1], several such proposals and results were discussed in diverse dimensions [2-24].

The focus of this Letter is the physics of one-dimensional defects in a CFT. Such defects can undergo nontrivial renormalization group flows while affecting the bulk very little far away from the defect. A few known examples of this kind include Wilson or 't Hooft lines in 4D gauge theories [25] and holography [26,27], symmetry defects and impurities in 3D quantum critical systems [28-33], etc. In two dimensions, line defects correspond to boundaries or interfaces and appear naturally as the low-energy limit of lattice systems with impurities (see, for instance, Refs. [34-38]).

There is already some extensive work on renormalization group flows on various defects [39-56]. For our purposes, it is important to highlight the conjecture of Affleck and

Published by the American Physical Society under the terms of the Creative Commons Attribution 4.0 International license. Further distribution of this work must maintain attribution to the author(s) and the published article's title, journal citation, and DOI. Funded by SCOAP.
Ludwig [39] for the decreasing entropy function on line defects in two dimensions and its subsequent proofs [42] and [48]. Here we will discuss the properties of line defects in arbitrary dimensions. We will define an entropy function and show that it monotonically decreases. In the Supplemental Material [57] we show how our result applies to a nontrivial flow between two different conformal Wilson lines in super Yang-Mills (SYM) theory in four dimensions.

The main idea we employ is that surrounding the line defect with conformal charges leads to nontrivial identifications in theory space when the defect is nonconformal. This can be expressed in terms of constraints on the dilaton living on the line defect. We show that these constraints translate to a monotonic entropy function.

DCFTs. - We consider local, reflection-positive Euclidean conformal field theories (CFTs) in $d \geq 2$ dimensions. We will be interested in CFTs in the presence of a line defect which preserves unitarity and locality. We will be interested in infinite straight lines or circular defects. At the fixed point of the (defect) renormalization group flow, the straight line defect preserves the subgroup $S L(2, \mathbb{R}) \times$ $S O(d-1)$ of the full conformal group. In this case the system is called a defect CFT (DCFT). In $d=2$, conformal line defects additionally preserve one copy of the Virasoro algebra.

DCFTs share many of the standard properties of CFTs. However, in general, the line defect does not support a stress tensor [58-60]. This statement really means that there is no possibility to localize energy on the line defect and energy always ends up being smeared into the bulk. The bulk stress tensor $T_{b}^{\mu \nu}$ obeys the following Ward identity (It is convenient to consider normalized correlation functions, so $\left\langle T_{b}^{\mu \nu}\right\rangle$ really stands for $\left\langle T_{b}^{\mu \nu} D\right\rangle /\langle D\rangle$, where $D$ is the defect operator.) $[47,59,61,62]$ :

$$
\nabla_{\mu} T_{b}^{\mu \nu}=-\delta_{D}^{d-1} n_{i}^{\nu} D^{i}
$$


where $\delta_{D}^{d-1}$ is a delta function localized at the defect, $\left\{n_{i}^{\nu}\right\}$ is a basis of $d-1$ unit vectors normal to the defect, and $D^{i}$ is the displacement operator $[47,59]$ (The defect $D$, and the displacement operator $D^{i}$, are distinguished by the superscript $i$.), which parametrizes the breaking of translations in the directions normal to the defect. Finally, we mention that all bulk correlation functions may be systematically decomposed into defect correlators via the bulk-to-defect OPE $[36,63]$. This allows us to study the DCFT data via a systematic bootstrap approach $[59,64,65]$, similar to the one usually adopted in standard CFTs [66-68].

It will be convenient for our purposes to consider the expectation of the $S L(2, \mathbb{R})$ charges wrapping the defect. These are obtained by integrating the stress tensor contracted with the appropriate Killing vector at a fixed distance $\varepsilon$ from the defect:

$$
Q_{\xi}(D)=\int_{\varepsilon} d^{d-1} \Sigma^{\mu}\left\langle T_{\mu \nu}^{b}\right\rangle \xi^{\nu}
$$

By conformal invariance we expect Eq. (2) to yield a vanishing result for both a straight line defect and a circular one. However, due to a subtlety with the action of conformal transformations on the point at infinity, for the straight line geometry the conformal charges vanish only when the distance between the integration surface and the defect diverges sufficiently fast as $x^{d} \rightarrow \pm \infty$ [25]. This issue is presumably related to the disagreement between the expectation value of circular and linear Maldacena-Wilson loops in $\mathcal{N}=4$ SYM [69-71]. We provide a detailed discussion regarding this subtlety in the Supplemental Material [57]. No issues of this sort arise for circular defects, hence we will focus on this geometry in what follows.

Let us consider for concreteness a circular defect of radius $R$ centered around the origin on the $\left(x^{1}, x^{2}\right)$ plane $x^{3}=\ldots=x^{d}=0$. The $S L(2, \mathbb{R})$ Killing vectors preserved by the circle are

$$
\begin{aligned}
& \xi_{(a)}^{\mu}=\frac{1}{2}\left[\delta_{a}^{\mu}\left(R+x^{2} / R\right)-2 x^{\mu} x_{a} / R\right], \\
& \xi_{(\phi)}^{\mu}=\delta_{a}^{\mu} \epsilon^{a b} x_{b},
\end{aligned}
$$

where $a=1,2$ and indices are raised or lowered with the Euclidean metric. Here, $\xi_{(a)}^{\mu}$ are linear combinations of translations and special conformal transformations on the defect plane, while $\xi_{(\phi)}^{\mu}$ generates rotations in the $\left(x^{1}, x^{2}\right)$ plane. In this geometry, there is no issue with the boundary condition at infinity and, consequently, the expectation values of the $S L(2, \mathbb{R})$ charges on a surface wrapping the defect (see Fig. 1) vanish,

$$
Q_{\xi}(D)=0 \quad(\text { circular defect })
$$

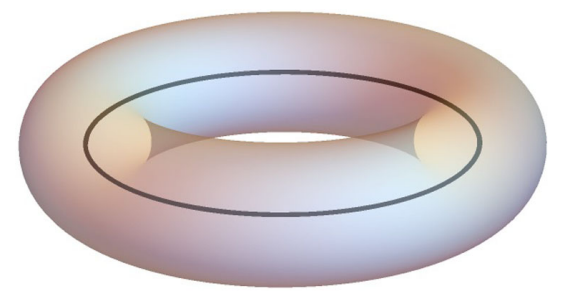

FIG. 1. An illustration of a toroidal surface wrapping a circular defect.

The statement (4) can be checked using the explicit form of the stress-tensor one-point function in a circular geometry, which depends on a unique constant [72] $h_{D}$ (see, e.g., Refs. [59,79] for the explicit expressions). In particular, in contrast to the infinite line, every point of the surface can be brought arbitrarily close to the defect compatibly with the identity (4) [80]. For this reason in the following we will focus on circular defects.

Defect $R G$.-The main goal of this work is to study defect renormalization group (DRG) flows. A DRG may be triggered perturbing a DCFT with one or more relevant defect operators. For instance, we may consider a defect operator $\mathcal{O}$ with $\Delta_{\mathcal{O}}<1$ :

$$
S_{\mathrm{DCFT}} \rightarrow S_{\mathrm{DCFT}}+M_{0}^{1-\Delta_{\mathcal{O}}} \int_{D} d \sigma \mathcal{O}(\sigma)
$$

where $\int_{D}$ stand for integration along the defect and $M_{0}$ is the mass scale of the flow. Conformal invariance [i.e., $S L(2, \mathbb{R})$ transformations that preserve the defect] is now explicitly broken by the scale $M_{0}$ to just translations along the defect.

Because of the locality of the bulk CFT, the bulk stress tensor remains conserved and traceless (up to possible bulk trace anomalies in curved space) away from the line. However, now a defect stress tensor $T_{D}$ is allowed. In other words, energy can now be stored on the defect. Not only $T_{D}$ is allowed, such an operator must always exist away from the fixed points of the defect. The existence of the operator $T_{D}$ is the reason that $S L(2, \mathbb{R})$ charges are no longer conserved. Since $T_{D}$ is localized to the defect, what we mean by saying that $S L(2, \mathbb{R})$ charges are no longer conserved is that, if the charges are integrated on surfaces that intersect the defect, then they are not invariant under small deformations.

Invariance under translations along the defect implies that Eq. (1) in the presence of $T_{D}$ is modified to

$$
\nabla_{\mu} T_{b}^{\mu \nu}=-\delta_{D}^{d-1} \dot{X}^{\nu} \dot{T}_{D}-\delta_{D}^{d-1} n_{i}^{\nu} D^{i}
$$

where $X^{\mu}(\sigma)$ is the embedding function describing the defect location and the dot stands for derivatives with respect to the line coordinate $\sigma$, so that $\dot{X}^{\nu}$ is a tangent vector to the defect. (Here we are assuming that the defect 
has a trivial induced submanifold metric $g_{D}=\dot{X}^{\mu} \dot{X}^{\nu} g_{\mu \nu}=$ 1 to simplify the notation.) Equation (6) merely expresses the energy balance between the bulk and the defect.

Spurion analysis and the dilaton.-As it often happens in the study of RG flows, it is useful to promote the renormalization group scale to a function of position $M(\sigma)=M_{0} e^{\Phi(\sigma)}[81,82]$, where $\Phi(\sigma)$ is a dimensionless background dilaton field. To linear order, the partition function of the theory depends on the dilaton through to the defect energy-momentum tensor [83]:

$$
\begin{aligned}
\left.\log Z\right|_{\Phi+\delta \Phi}= & \left.\log Z\right|_{\Phi}+\int_{D} d \sigma \delta \Phi(\sigma)\left\langle T_{D}(\sigma)\right\rangle_{\Phi} \\
& +\frac{1}{2} \int_{D} d \sigma_{1} \int_{D} d \sigma_{2} \delta \Phi\left(\sigma_{1}\right) \delta \Phi\left(\sigma_{2}\right) \\
& \times\left\langle T_{D}\left(\sigma_{1}\right) T_{D}\left(\sigma_{2}\right)\right\rangle_{\Phi}+\ldots
\end{aligned}
$$

The background dilaton field acts a source for the theory. This in turn modifies the conservation equation (6) as follows $[61,62]$ :

$$
\nabla_{\mu} T_{b}^{\mu \nu}=-\delta_{D}^{d-1} \dot{X}^{\nu}\left(\dot{T}_{D}-\dot{\Phi} T_{D}\right)-\delta_{D}^{d-1} n_{i}^{\nu} D^{i} .
$$

If one views the coordinate along the defect as time, then a nontrivial $\Phi(\sigma)$ renders the theory time dependent and (8) relates the nonconservation rate of the charge associated with translations along the defect with the derivative of the dilaton source.

A position-dependent mass scale breaks the $S L(2, \mathbb{R})$ symmetry completely. What we gain by introducing the general background field $\Phi(\sigma)$ is that $S L(2, \mathbb{R})$ allows us to relate different theories instead of directly placing constraints on a given theory. Indeed, we will use Eq. (8) in what follows to derive some nontrivial identities relating theories with different values for the source $\Phi(\sigma)$.

$R G$ flows induced by the broken charges. - It is crucial to realize that the identity (4) holds irrespectively of the breaking of scale invariance on the defect [i.e., it holds for any $\Phi(\sigma)]$. This is because the charges wrapping the defect do not intersect it and hence such charges are oblivious to what happens on the defect and they remain invariant under small deformations. They can be moved off to infinity where they annihilate the vacuum. (To see that, one can realize the wrapping surface as the difference between two $S^{d-1}$ surfaces outside and inside the loop.)

As we explained, on general grounds one expects that $S L(2, \mathbb{R})$ transformation can be reabsorbed into a transformation of the dilaton $\Phi(\sigma)$, leading to relations between different theories. This can be made precise by using Eq. (4). To that end, consider shrinking the radius of the topological surface enclosing the defect (see Fig. 1). It is clear from Gauss's law that the only contribution in the integration of the stress tensor arises from the right-hand side in Eq. (8). We therefore conclude that Eq. (4) implies the following relation [84]:

$$
\begin{aligned}
0=Q_{\xi}(D) & =\int d^{d-1} \Sigma^{\mu}\left\langle T_{\mu \nu}^{b}\right\rangle \xi^{\nu} \\
& =\int_{D} d \sigma\left(\dot{\xi}_{D}+\xi_{D} \dot{\Phi}\right)\left\langle T_{D}\right\rangle,
\end{aligned}
$$

where in the second line we integrated by parts and we denoted by $\xi_{D}$ the projection of the Killing vectors (3) on the defect. We crucially used the fact that the normal components of the $S L(2, \mathbb{R})$ Killing vectors vanish on the defect. In fact, for more general conformal Killing vectors which do not leave the loop invariant, an analogous identity picks an additional contribution from the displacement operator in Eq. (8) but we do not study these identities here.

Because of the linear coupling between the defect stress tensor and the dilaton, we may interpret Eq. (9) as an equivalence between defects with different DRG scales $M(\sigma)$ :

$$
\Phi \sim \Phi+\alpha\left(\dot{\xi}_{D}+\xi_{D} \dot{\Phi}\right) \quad|\alpha| \ll 1,
$$

for any $S L(2, \mathbb{R})$ Killing vector $\xi$ and any infinitesimal $\alpha$. This observation is most useful when considering the expansion of the partition function (7) around $\Phi=0$. Demanding the equivalence (10) at each order in the field expansion we then find an infinite number of identities for the correlation functions of the defect stress tensor. At second order in the field expansion we obtain the following one (omitting the subscript $\Phi=0$ from now on):

$$
\begin{aligned}
& \int_{D} d \sigma \xi_{D}(\sigma) \dot{\Phi}(\sigma)\left\langle T_{D}(\sigma)\right\rangle \\
& \quad=-\int_{D} d \sigma_{1} \int_{D} d \sigma_{2} \dot{\xi}_{D}\left(\sigma_{1}\right) \Phi\left(\sigma_{2}\right)\left\langle T_{D}\left(\sigma_{1}\right) T_{D}\left(\sigma_{2}\right)\right\rangle .
\end{aligned}
$$

Crucially, this identity holds for any $\Phi(\sigma)$. Notice that the right-hand side of Eq. (11) for generic choices of the dilaton profile is naively divergent. Our arguments however ensure that these identities must hold in any regularization scheme which preserves the invariance of the partition function under diffeomorphisms and defect reparametrizations.

At this point it is useful to specify a cylindrical system of coordinates on the defect: $x^{1}=R \cos \phi, x^{2}=R \sin \phi$ and set $\sigma=R \phi$. The projection of the three Killing vectors in Eq. (3) reads, respectively,

$$
\xi_{D}=-\sin \phi, \quad \xi_{D}=\cos \phi, \quad \xi_{D}=-1 .
$$

Equation (11) is trivial for $\xi_{D}=-1$, but provides nontrivial constraints for the other two choices, which lead to identical constraints. A particularly useful relation is obtained choosing $\xi_{D}=-\sin \phi$ and $\Phi \propto \cos \phi$ in Eq. (11). This leads to

$$
\begin{aligned}
& R \int_{D} d \phi\left\langle T_{D}(\phi)\right\rangle \\
& =R^{2} \int_{D} d \phi_{1} \int_{D} d \phi_{2}\left\langle T_{D}\left(\phi_{1}\right) T_{D}\left(\phi_{2}\right)\right\rangle \cos \left(\phi_{1}-\phi_{2}\right),
\end{aligned}
$$


where we used trigonometric identities and invariance under translations along the defect to simplify both sides. Equation (13) will be very useful in providing a gradient formula for the DRG flow of a suitably defined defectentropy.

The defect entropy.-Our discussion thus far focused on defects in flat space, but all our considerations apply on all conformally equivalent manifolds. These include the $d$ dimensional sphere of radius $R$, with the defect spanning a maximal circle, and the cylinder $\mathbb{R} \times S^{d-1}$, with the defect on the equator of $S^{d-1}$ at a fixed value of the Euclidean time $\tau=\log x^{2} / R=0$.

We can use any of these geometries to define a defect $g$ function, $g\left(M_{0} R\right)$, in terms of the partition function in the presence of the defect, normalized by the partition function without it:

$$
\log g\left(M_{0} R\right)=\log Z_{\mathcal{M}}-\log Z_{\mathcal{M}}^{(C F T)},
$$

where $\log Z_{\mathcal{M}}^{(C F T)}$ is the partition function of the theory without the defect [85]. The defect contribution $g$ depends only on the dimensionless product $M_{0} R$ and it reduces to a constant at the fixed points (in a sense that we will explain below).

We must now ask to what extent is $g$ well defined at the fixed points and away from them. $\log g$ can be shifted by the addition of a cosmological constant counterterm $\int d \sigma M_{0} \sim$ $M_{0} R$ with an arbitrary coefficient. All other nontrivial geometric invariants which are analytic around the flat metric have dimension larger than 1 and cannot appear as counterterms. Therefore no additional ambiguities exist in $d>2$ (we will discuss $d=2$ more in detail below). Therefore, one can obtain a scheme-independent quantity which we will refer to as the defect entropy, defined as [86]

$$
s\left(M_{0} R\right)=\left(1-R \frac{\partial}{\partial R}\right) \log g\left(M_{0} R\right) .
$$

At the fixed points, $s\left(M_{0} R\right)$ is a pure number which is scheme independent. It is equal to the perimeter-independent contribution to $\log g\left(M_{0} R\right)$ at the fixed point. We will refer to these fixed point values of $g$ as $g_{\mathrm{UV}}, g_{\mathrm{IR}}$, respectively. We will show that $s\left(M_{0} R\right)$ decreases monotonically under DRG, implying $g_{\mathrm{UV}}>g_{\mathrm{IR}}$.

In $d=2$ Eq. (15) coincides with the interface contribution to the thermal entropy of the theory. To make the connection with $d=2$ precise, one needs to remember that in $d=2$ we can also allow the counterterm $\int d \sigma K$, where $K$ is the extrinsic curvature [88]. Such a term vanishes for a maximal circle in $S^{2}$ and on $\mathbb{R} \times S^{1}$ and therefore all our conclusions hold unaltered on those manifolds. Furthermore $C P T$ invariance implies that the coefficient of this counterterm should be purely imaginary. Therefore the definition in Eq. (15) is meaningful also in flat space provided we focus on the real part of the defect entropy.
The gradient formula.-We now have all the ingredients to derive a gradient formula for the DRG flow of the defect entropy. Since $g$ depends on $M_{0} R$ only, for constant dilaton $\Phi$, it follows that $g$ depends on the combination $R M_{0} e^{\Phi}$. We may therefore write the variation of the defect entropy $s$ under a change in the mass scale as follows:

$M_{0} \frac{\partial}{\partial M_{0}} s\left(M_{0} R\right)=\left[\left(\frac{d}{d \Phi}-\frac{d^{2}}{d \Phi^{2}}\right) \log g\left(R M_{0} e^{\Phi}\right)\right]_{\Phi=0}$.

Using the expansion (7) for constant $\Phi$ we then can write Eq. (16) in terms of correlation functions of the defect stress tensor

$$
\begin{aligned}
M_{0} \frac{\partial}{\partial M_{0}} s\left(M_{0} R\right)= & R \int_{D} d \phi\left\langle T_{D}(\phi)\right\rangle \\
& -R^{2} \int_{D} d \phi_{1} \int_{D} d \phi_{2}\left\langle T_{D}\left(\phi_{1}\right) T_{D}\left(\phi_{2}\right)\right\rangle .
\end{aligned}
$$

Equation (17) may not seem very useful at first sight. It is not manifestly sign definite, nor is it manifestly finite. To clarify these issues, we can rewrite the first term using Eq. (13). We obtain

$$
\begin{aligned}
& M_{0} \frac{\partial s}{\partial M_{0}} \\
& =-R^{2} \int_{D} d \phi_{1} \int_{D} d \phi_{2}\left\langle T_{D}\left(\phi_{1}\right) T_{D}\left(\phi_{2}\right)\right\rangle\left[1-\cos \left(\phi_{1}-\phi_{2}\right)\right] .
\end{aligned}
$$

The right-hand side of Eq. (18) is free of divergences and ambiguities due to the double zero of $1-\cos \left(\phi_{1}-\phi_{2}\right)$. Furthermore, (18) is manifestly negative in a reflection positive theory (note that this also applies to a connected 2-point function, as on the right-hand side of Eq. (18)). Therefore, we deduce that $s$ monotonically decreases along defect RG flows, implying that the UV and IR DCFT satisfy

$$
g_{\mathrm{UV}}>g_{\mathrm{IR}}
$$

Equation (18) additionally implies that $s$ does not depend on the marginal parameters on the defect [91].

In $d=2$, Eq. (19) was originally conjectured to hold for boundaries (and therefore, using the folding trick, for interfaces) by Affleck and Ludwig [37,39]. In $d=2$, in the regime where the DRG flow can be described in terms of finitely many couplings and beta functions, a gradient formula equivalent to Eq. (18) was proposed in the context of string field theory [98-102]. It was then established by Friedan and Konechny [42]. An alternative proof of Eq. (19) in $d=2$ was also given [48] using quantum information methods. (See, for instance, also Refs. [41,44,103] for a holographic setup.) Our work provides an extension of those results to line defects in an arbitrary number of 
dimensions. We also remark that the inequality (19) was recently conjectured in Ref. [50] for arbitrary $d$. Another remark is that the trivial line has $g=1$. However, it may a priori be that $g<1$ for some nontrivial lines, as sometimes happens in two dimensions $[104,105]$.

Equation (19) was extensively checked in $d=2$, see, e.g., Refs. [37-39,106]. We additionally verified our results (18) and (19) in several concrete examples, including a flow between Wilson lines in $\mathcal{N}=4 \mathrm{SYM}$ previously studied in Refs. [27,107]. Details can be found in the Supplemental Material [57].

Finally, we remark that the partition function of higherdimensional defects is subject to further ambiguities besides a cosmological constant, rendering a generalization of our arguments not straightforward. For two- and fourdimensional defects irreversibility of the DRG flow was proven via different means, using Weyl anomaly matching $[47,55]$.

We acknowledge useful discussions with Bartolomeu Fiol, Sergei Gukov, Simeon Hellerman, Márk Mezei, Luigi Tizzano, Cumrun Vafa, and Yifan Wang. G. C. is supported by the Simons Foundation (Simons Collaboration on the Non-perturbative Bootstrap) Grants No. 488647 and No. 397411. Z. K. and A. R. M. are supported in part by the Simons Foundation Grant No. 488657 (Simons Collaboration on the Non-Perturbative Bootstrap) and the BSF Grant No. 2018204. The work of A. R. M. was also supported in part by the Zuckerman-CHE STEM Leadership Program.

*gcuomo@scgp.stonybrook.edu †komargodski@scgp.stonybrook.edu *araviv-moshe@scgp.stonybrook.edu

[1] A. B. Zomolodchikov, "Irreversibility" of the flux of the renormalization group in a $2 \mathrm{D}$ field theory, Sov. J. Exp. Theor. Phys. Lett. 43, 730 (1986).

[2] J. L. Cardy, Is there a c theorem in four-dimensions?, Phys. Lett. B 215, 749 (1988).

[3] A. Cappelli, D. Friedan, and J. I. Latorre, C theorem and spectral representation, Nucl. Phys. B352, 616 (1991).

[4] H. Osborn, Weyl consistency conditions and a local renormalization group equation for general renormalizable field theories, Nucl. Phys. B363, 486 (1991).

[5] R. C. Myers and A. Sinha, Holographic c-theorems in arbitrary dimensions, J. High Energy Phys. 01 (2011) 125.

[6] D. L. Jafferis, I. R. Klebanov, S. S. Pufu, and B. R. Safdi, Towards the F-theorem: $N=2$ field theories on the threesphere, J. High Energy Phys. 06 (2011) 102.

[7] Z. Komargodski and A. Schwimmer, On renormalization group flows in four dimensions, J. High Energy Phys. 12 (2011) 099.

[8] H. Casini and M. Huerta, On the RG running of the entanglement entropy of a circle, Phys. Rev. D 85, 125016 (2012).

[9] H. Elvang, D. Z. Freedman, L.-Y. Hung, M. Kiermaier, R. C. Myers, and S. Theisen, On renormalization group flows and the a-theorem in 6d, J. High Energy Phys. 10 (2012) 011.

[10] H. Elvang and T. M. Olson, RG flows in d dimensions, the dilaton effective action, and the a-theorem, J. High Energy Phys. 03 (2013) 034.

[11] K. Yonekura, Perturbative c-theorem in d-dimensions, J. High Energy Phys. 04 (2013) 011.

[12] O. Antipin, M. Gillioz, E. Mølgaard, and F. Sannino, The a theorem for gauge-Yukawa theories beyond Banks-Zaks fixed point, Phys. Rev. D 87, 125017 (2013).

[13] B. Grinstein, A. Stergiou, and D. Stone, Consequences of Weyl consistency conditions, J. High Energy Phys. 11 (2013) 195.

[14] I. Jack and H. Osborn, Constraints on RG flow for four dimensional quantum field theories, Nucl. Phys. B883, 425 (2014).

[15] F. Baume, B. Keren-Zur, R. Rattazzi, and L. Vitale, The local Callan-Symanzik equation: Structure and applications, J. High Energy Phys. 08 (2014) 152.

[16] B. Grinstein, D. Stone, A. Stergiou, and M. Zhong, Challenge to the $a$ Theorem in Six Dimensions, Phys. Rev. Lett. 113, 231602 (2014).

[17] S. Giombi and I. R. Klebanov, Interpolating between $a$ and F, J. High Energy Phys. 03 (2015) 117.

[18] I. Jack, D. R. T. Jones, and C. Poole, Gradient flows in three dimensions, J. High Energy Phys. 09 (2015) 061.

[19] C. Cordova, T. T. Dumitrescu, and K. Intriligator, Anomalies, renormalization group flows, and the atheorem in six-dimensional $(1,0)$ theories, J. High Energy Phys. 10 (2016) 080.

[20] H. Casini, M. Huerta, R. C. Myers, and A. Yale, Mutual information and the F-theorem, J. High Energy Phys. 10 (2015) 003.

[21] S. S. Pufu, The F-theorem and F-maximization, J. Phys. A 50, 443008 (2017).

[22] H. Casini, E. Testé, and G. Torroba, Markov Property of the Conformal Field Theory Vacuum and the a Theorem, Phys. Rev. Lett. 118, 261602 (2017).

[23] M. Fluder and C.F. Uhlemann, Evidence for a $5 \mathrm{~d}$ F-theorem, J. High Energy Phys. 02 (2021) 192.

[24] L. V. Delacretaz, A. L. Fitzpatrick, E. Katz, and M. T. Walters, Thermalization and hydrodynamics of twodimensional quantum field theories, arXiv:2105.02229.

[25] A. Kapustin, Wilson-'t Hooft operators in fourdimensional gauge theories and S-duality, Phys. Rev. D 74, 025005 (2006).

[26] J. Gomis and F. Passerini, Holographic Wilson loops, J. High Energy Phys. 08 (2006) 074

[27] J. Polchinski and J. Sully, Wilson loop renormalization group flows, J. High Energy Phys. 10 (2011) 059.

[28] M. Billó, M. Caselle, D. Gaiotto, F. Gliozzi, M. Meineri, and R. Pellegrini, Line defects in the $3 \mathrm{~d}$ Ising model, J. High Energy Phys. 07 (2013) 055.

[29] D. Gaiotto, D. Mazac, and M. F. Paulos, Bootstrapping the 3d Ising twist defect, J. High Energy Phys. 03 (2014) 100.

[30] S. Giombi, E. Helfenberger, Z. Ji, and H. Khanchandani, Monodromy defects from hyperbolic space, arXiv: 2102.11815.

[31] S. Sachdev, C. Buragohain, and M. Vojta, Quantum impurity in a nearly critical two-dimensional antiferromagnet, Science 286, 2479 (1999). 
[32] M. Vojta, C. Buragohain, and S. Sachdev, Quantum impurity dynamics in two-dimensional antiferromagnets and superconductors, Phys. Rev. B 61, 15152 (2000).

[33] S. Liu, H. Shapourian, A. Vishwanath, and M. A. Metlitski, Magnetic impurities at quantum critical points: Large- $N$ expansion and SPT connections, Phys. Rev. B 104, 104201 (2021).

[34] A. Tsvelick and P. Wiegmann, Exact solution of the multichannel kondo problem, scaling, and integrability, J. Stat. Phys. 38, 125 (1985).

[35] N. Ishibashi, The boundary and crosscap states in conformal field theories, Mod. Phys. Lett. A 04, 251 (1989).

[36] J. L. Cardy, Boundary conditions, fusion rules and the Verlinde formula, Nucl. Phys. B324, 581 (1989).

[37] I. Affleck and A. W. W. Ludwig, Exact conformal-fieldtheory results on the multichannel Kondo effect: Singlefermion Green's function, self-energy, and resistivity, Phys. Rev. B 48, 7297 (1993).

[38] I. Affleck, Conformal field theory approach to the Kondo effect, Acta Phys. Pol. B 26, 1869 (1995).

[39] I. Affleck and A. W. W. Ludwig, Universal Noninteger 'Ground State Degeneracy' in Critical Quantum Systems, Phys. Rev. Lett. 67, 161 (1991).

[40] P. Dorey, I. Runkel, R. Tateo, and G. Watts, g-Function Flow in Perturbed Boundary Conformal Field Theories, Nucl. Phys. B578, 85 (2000).

[41] S. Yamaguchi, Holographic RG flow on the defect and $g$ theorem, J. High Energy Phys. 10 (2002) 002.

[42] D. Friedan and A. Konechny, On the Boundary Entropy of One-Dimensional Quantum Systems at Low Temperature, Phys. Rev. Lett. 93, 030402 (2004).

[43] T. Azeyanagi, A. Karch, T. Takayanagi, and E. G. Thompson, Holographic calculation of boundary entropy, J. High Energy Phys. 03 (2008) 054.

[44] T. Takayanagi, Holographic Dual of BCFT, Phys. Rev. Lett. 107, 101602 (2011).

[45] J. Estes, K. Jensen, A. O'Bannon, E. Tsatis, and T. Wrase, On holographic defect entropy, J. High Energy Phys. 05 (2014) 084.

[46] D. Gaiotto, Boundary F-maximization, arXiv:1403.8052.

[47] K. Jensen and A. O'Bannon, Constraint on Defect and Boundary Renormalization Group Flows, Phys. Rev. Lett. 116, 091601 (2016).

[48] H. Casini, I. Salazar Landea, and G. Torroba, The gtheorem and quantum information theory, J. High Energy Phys. 10 (2016) 140.

[49] N. Andrei et al., Boundary and defect CFT: Open problems and applications, J. Phys. A 53, 453002 (2020).

[50] N. Kobayashi, T. Nishioka, Y. Sato, and K. Watanabe, Towards a $C$-theorem in defect CFT, J. High Energy Phys. 01 (2019) 039.

[51] H. Casini, I. Salazar Landea, and G. Torroba, Irreversibility in quantum field theories with boundaries, J. High Energy Phys. 04 (2019) 166.

[52] S. Giombi and H. Khanchandani, CFT in AdS and boundary RG flows, J. High Energy Phys. 11 (2020) 118.

[53] Y. Wang, Surface defect, anomalies and $b$-extremization, J. High Energy Phys. 11 (2021) 122.
[54] T. Nishioka and Y. Sato, Free energy and defect $C$-theorem in free scalar theory, J. High Energy Phys. 05 (2021) 074.

[55] Y. Wang, Defect $a$-theorem and $a$-maximization, arXiv: 2101.12648.

[56] Y. Sato, Free energy and $\operatorname{defect} C$-theorem in free fermion, J. High Energy Phys. 05 (2021) 202.

[57] See Supplemental Material at http://link.aps.org/ supplemental/10.1103/PhysRevLett.128.021603 for examples and details on the difference between circular and straight line defects.

[58] Y. Nakayama, Is boundary conformal in CFT?, Phys. Rev. D 87, 046005 (2013).

[59] M. Billò, V. Gonçalves, E. Lauria, and M. Meineri, Defects in conformal field theory, J. High Energy Phys. 04 (2016) 091.

[60] C. P. Herzog and K.-W. Huang, Boundary conformal field theory and a boundary central charge, J. High Energy Phys. 10 (2017) 189.

[61] H. Osborn and A. C. Petkou, Implications of conformal invariance in field theories for general dimensions, Ann. Phys. (N.Y.) 231, 311 (1994).

[62] G. Cuomo, M. Mezei, and A. Raviv-Moshe, Boundary conformal field theory at large charge, J. High Energy Phys. 10 (2021) 143.

[63] F. Gliozzi, P. Liendo, M. Meineri, and A. Rago, Boundary and Interface CFTs from the conformal bootstrap, J. High Energy Phys. 05 (2015) 036.

[64] P. Liendo, L. Rastelli, and B. C. van Rees, The bootstrap program for boundary $\mathrm{CFT}_{d}$, J. High Energy Phys. 07 (2013) 113.

[65] E. Lauria, P. Liendo, B. C. Van Rees, and X. Zhao, Line and surface defects for the free scalar field, J. High Energy Phys. 01 (2021) 060.

[66] A. A. Belavin, A. M. Polyakov, and A. B. Zamolodchikov, Infinite conformal symmetry in two-dimensional quantum field theory, Nucl. Phys. B241, 333 (1984).

[67] R. Rattazzi, V.S. Rychkov, E. Tonni, and A. Vichi, Bounding scalar operator dimensions in 4D CFT, J. High Energy Phys. 12 (2008) 031.

[68] D. Poland, S. Rychkov, and A. Vichi, The conformal bootstrap: Theory, numerical techniques, and applications, Rev. Mod. Phys. 91, 015002 (2019).

[69] J. K. Erickson, G. W. Semenoff, and K. Zarembo, Wilson loops in $\mathrm{N}=4$ supersymmetric Yang-Mills theory, Nucl. Phys. B582, 155 (2000).

[70] N. Drukker and D. J. Gross, An exact prediction of $N=4$ SUSYM theory for string theory, J. Math. Phys. (N.Y.) 42, 2896 (2001).

[71] V. Pestun, Localization of gauge theory on a four-sphere and supersymmetric Wilson loops, Commun. Math. Phys. 313 (2012) 71.

[72] The coefficient $h_{D}$ is a physical characteristic of the DCFT and it was computed in various supersymmetric examples [73-77] (for a general approach to supersymmetric line defects see Ref. [78]).

[73] D. Correa, J. Henn, J. Maldacena, and A. Sever, An exact formula for the radiation of a moving quark in $\mathrm{N}=4$ super Yang Mills, J. High Energy Phys. 06 (2012) 048. 
[74] F. Fucito, J. F. Morales, and R. Poghossian, Wilson loops and chiral correlators on squashed spheres, J. High Energy Phys. 11 (2015) 064.

[75] B. Fiol, E. Gerchkovitz, and Z. Komargodski, Exact Bremsstrahlung Function in $N=2$ Superconformal Field Theories, Phys. Rev. Lett. 116, 081601 (2016).

[76] L. Bianchi, M. Lemos, and M. Meineri, Line Defects and Radiation in $\mathcal{N}=2$ Conformal Theories, Phys. Rev. Lett. 121, 141601 (2018).

[77] L. Bianchi, M. Billò, F. Galvagno, and A. Lerda, Emitted radiation and geometry, J. High Energy Phys. 01 (2020) 075.

[78] N. B. Agmon and Y. Wang, Classifying superconformal defects in diverse dimensions Part I: Superconformal lines, arXiv:2009.06650.

[79] J. Gomis, S. Matsuura, T. Okuda, and D. Trancanelli, Wilson loop correlators at strong coupling: From matrices to bubbling geometries, J. High Energy Phys. 08 (2008) 068.

[80] In spite of this, such a configuration is conformally equivalent to a straight line surrounded by a surface whose radius becomes increasingly large as the line extends to infinity.

[81] Z. Komargodski, The constraints of conformal symmetry on RG flows, J. High Energy Phys. 07 (2012) 069.

[82] M. A. Luty, J. Polchinski, and R. Rattazzi, The $a$-theorem and the asymptotics of 4D quantum field theory, J. High Energy Phys. 01 (2013) 152.

[83] In general, the conformal symmetry of the line defect is violated by a nonvanishing $T_{D}$. Such a nonvanishing $T_{D}$ can be split (not unambiguously) into a $c$ number due to conformal anomalies and a nontrival local operator. A somewhat common convention, which we also adopt here, is to have the dilaton couplings compensate for the operatorial violation of scale invariance, but not the trace anomalies, which might generically be present in the bulk-see, e.g., [47,81] for details.

[84] We use conventions such that the bulk stress tensor $T_{b}^{\mu \nu}$ does not contain any contribution proportional to $\delta_{D}^{d-1}$ and therefore it is traceless everywhere $[47,62]$.

[85] $\log Z_{\mathcal{M}}^{(C F T)}$ is divergent on the infinite cylinder or in flat space, moreover, in general, it is ambiguous due to various counterterms. But these bulk issues cancel from the definition of $g\left(M_{0} R\right)$.

[86] This terminology differs from that of Ref. [50], where the term defect entropy referred to the defect contribution to the entanglement entropy. Note that in $d=2$ the defect entanglement entropy and ordinary entropy coincide at fixed points because $h_{D}=0$. Here we see that the correct generalization to higher dimensions involves the defect entropy and not the defect entanglement entropy which is also sensitive to $h_{D}$ [87].

[87] A. Lewkowycz and J. Maldacena, Exact results for the entanglement entropy and the energy radiated by a quark, J. High Energy Phys. 05 (2014) 025.

[88] For $d>2$ the extrinsic curvature is not analytic at zero and therefore is not an allowed counterterm; see, e.g., Ref. [47] for a concise review of submanifold geometry. The counterterm $\int d \sigma K$ was discussed in a different context when studying the entanglement entropy in $(2+1)$ dimensions, see, e.g., Refs. [89,90].

[89] T. Grover, A. M. Turner, and A. Vishwanath, Entanglement entropy of gapped phases and topological order in three dimensions, Phys. Rev. B 84, 195120 (2011).

[90] H. Liu and M. Mezei, A refinement of entanglement entropy and the number of degrees of freedom, J. High Energy Phys. 04 (2013) 162.

[91] It is often the case that $g$ and $s$ depend on the marginal couplings of the bulk CFT [92-96]. Furthermore $g$ and $s$ do not have any obvious monotonicity property under bulk RG flows [97].

[92] S. Elitzur, E. Rabinovici, and G. Sarkissian, On least action D-branes, Nucl. Phys. B541, 246 (1999).

[93] S. Fredenhagen, M. R. Gaberdiel, and C. A. Keller, Bulk induced boundary perturbations, J. Phys. A 40, F17 (2007).

[94] S. Elitzur, B. Karni, and E. Rabinovici, Induced boundary flow on the $\mathrm{c}=1$ orbifold moduli space, J. Phys. A 45, 455401 (2012).

[95] L. Bianchi, Marginal deformations and defect anomalies, Phys. Rev. D 100, 126018 (2019).

[96] C. P. Herzog and I. Shamir, How a-Type Anomalies can Depend on Marginal Couplings, Phys. Rev. Lett. 124, 011601 (2020).

[97] D. R. Green, M. Mulligan, and D. Starr, Boundary entropy can increase under bulk RG flow, Nucl. Phys. B798, 491 (2008).

[98] E. Witten, On background independent open string field theory, Phys. Rev. D 46, 5467 (1992).

[99] E. Witten, Some computations in background independent off-shell string theory, Phys. Rev. D 47, 3405 (1993).

[100] S. L. Shatashvili, Comment on the background independent open string theory, Phys. Lett. B 311, 83 (1993).

[101] S. L. Shatashvili, On the problems with background independence in string theory, Alg. Anal. 6, 215 (1994).

[102] D. Kutasov, M. Marino, and G. W. Moore, Some exact results on tachyon condensation in string field theory, J. High Energy Phys. 10 (2000) 045.

[103] J. Erdmenger, C. Hoyos, A. O'Bannon, and J. Wu, A holographic model of the Kondo effect, J. High Energy Phys. 12 (2013) 086.

[104] M. Oshikawa and I. Affleck, Boundary conformal field theory approach to the critical two-dimensional Ising model with a defect line, Nucl. Phys. B495, 533 (1997).

[105] M. Oshikawa and I. Affleck, Defect Lines in the Ising Model and Boundary States on Orbifolds, Phys. Rev. Lett. 77, 2604 (1996).

[106] A. Konechny, g function in perturbation theory, Int. J. Mod. Phys. A 19, 2545 (2004).

[107] M. Beccaria, S. Giombi, and A. Tseytlin, Non-supersymmetric Wilson loop in $\mathcal{N}=4 \mathrm{SYM}$ and defect $1 \mathrm{~d}$ CFT, J. High Energy Phys. 03 (2018) 131. 